\title{
RESPONSE TO GEFITINIB IN PERICARDIAL EFFUSION DUE TO LUNG CANCER
}

\author{
Norihiro Kikuchi, Hiroaki Satoh, Takahide Kodama, Norihiro Haraguchi, Kiyohisa Sekizawa
}

University of Tsukuba, Institute of Clinical Medicine: Division of Respiratory Medicine

Summary: We described a 70 years old patient with pericardial effusion due to adenocarcinoma of the lung, in whom gefitinib, which is an oral selective inhibitor of the epidermal growth factor receptor of tyrosine kinase, demonstrated a marked antitumor effect. We recommend possible consideration of a treatment with gefitinib for female patients with pericarditis carcinomatosa due to lung adenocarcinoma, even if they have a poor performance status and are not indicated for other intensive therapy.

Key words: Lung cancer; Gefitinib; Pericardial effusion

\section{Introduction}

Gefitinib is an oral selective inhibitor of the epidermal growth factor receptor (EGFR) tyrosine kinase, and is regarded as a safe agent, although some toxic effects such as interstitial pneumonia, diarrhea and skin rash have been recognized $(3,4,5)$. We present a female patient with pericardial effusion due to adenocarcinoma of the lung, in whom gefitinib demonstrated a marked antitumor effect.

\section{Case report}

A 70-year-old woman was diagnosed with metastatic adenocarcinoma of the lung when she was presented with supraclavicular and cervical lymph node swelling. Chest CT scan demonstrated a pericardial effusion (Fig. 1). Chest CT scan also showed a $3.5 \times 2.5 \mathrm{~cm}$ right lung mass with mediastinal lymph node swelling. Pathology samples from cervical lymph nodes confirmed adenocarcinoma of the lung. Because of decreasing performance status of the patient, she received $250 \mathrm{mg}$ gefitinib once daily. Physical examination on the day-10 of gefitinib administration demonstrated marked regression of the supraclavicular and cervical lymph node swelling. A chest radiograph on day- 28 revealed regression of pericardial effusion. Chest CT scan on day- 100 showed disappearance of pericardial effusion (Fig. 2). The lung mass and mediastinal lymph node swelling have also disappeared. Adverse effects considered to be possibly due to gefitinib administration including interstitial pneumonia, skin rash, and diarrhea were not observed. Patient is currently active and able to perform her activity of daily living independently. She continues to take gefitinib daily. This effect lasted for 6 months.

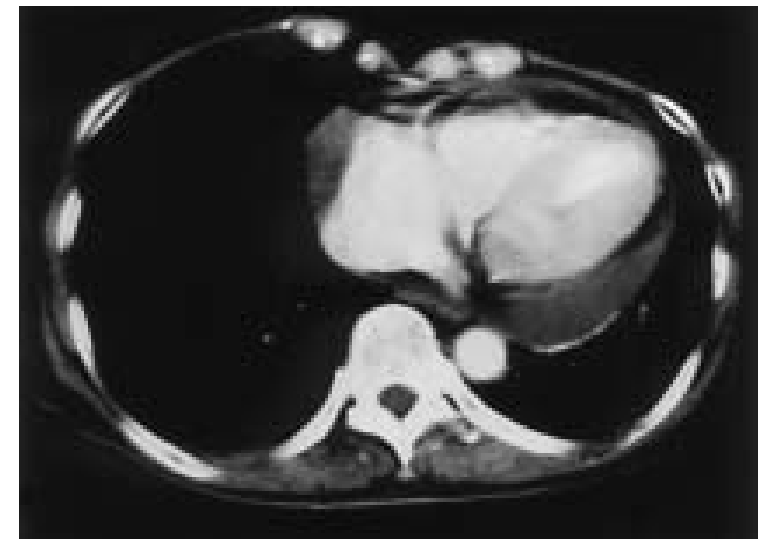

Fig. 1: Chest CT scan on admission showing a pericarcial effusion.

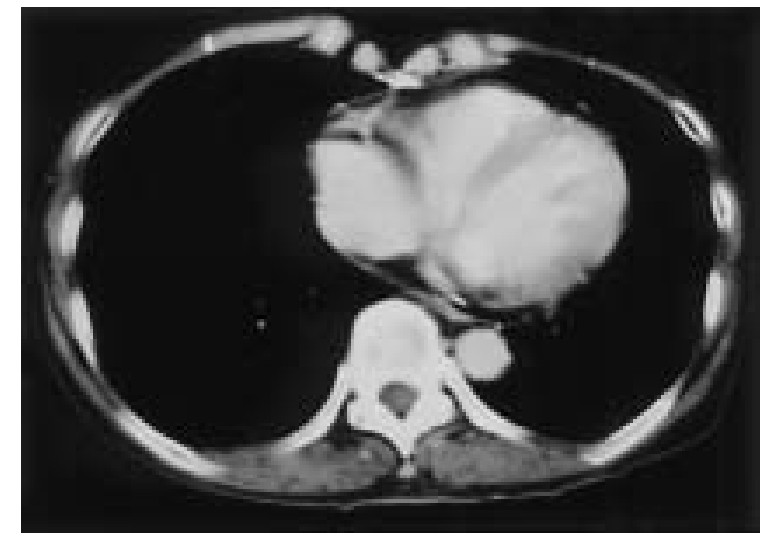

Fig. 2: Chest CT scan on day-100 of gefitinib administration showing disappearance of pericardial effusion. 


\section{Discussion}

EGFR is expressed in a wide range of solid tumors including non-small cell lung cancer (NSCLC), and gefitinib is a selective, orally administrated EGFR tyrosine kinase inhibitor that causes complete inhibition of EGF-stimulated EGFR autophosphorylation in cell lines at submicromolar concentrations (3). Two phase II studies assessing the efficacy and safety of gefitinib in patients with NSCLC have been reported with encouraging results, showing significant activity against NSCLC $(3,5)$. We administrated gefitinib to a patient with pericarditis carcinomatosa due to lung adenocarcinoma. Although the patient had a poor performance status, a remarkable antitumor activity was obtained without any serious adverse effects. Both female gender and adenocarcinoma are identified as favorable prognostic factor in a multivariate analysis of patients in the previous randomized double blind trials $(3,5)$. Our patient was a woman diagnosed with adenocarcinoma of the lung, and as such, might have two advantages of gender and histology, although she had poor performance status. Very recent reports showed dramatic effect of gefitinib in patients with poor performance status $(2,6)$. These two patients were also female gender and adenocarcinoma of the lung $(2,6)$. One of the patients also had a pericardial effusion and bilateral pleural effusion, which decreased on day-10 of gefitinib administration started. Together with the result of our patient, we recommend possible consideration of a treatment with gefitinib for female patients with pericarditis car- cinomatosa due to lung adenocarcinoma, even if they have a poor peroformance status and are not indicated for other intensive therapy.

\section{References}

1. Ciargiello F, Caputo R, Bianco R et al. Antitumor effect and potentiation of cytotoxic drugs activity in human cancer cells by ZD-1839 (Iressa), an epidermal growth factor receptor-selective tyrosine kinase inhibitor. Clin Cancer Res 2000; 6:2053-63.

2. Fujiwara K, Kiura K, Ueoka H et al. Dramatic effect of ZD1839 ('Iressa') in a patient with advanced non-small-cell lung cancer and poor performance status. Lung Cancer 2003;40:73-6.

3. Fukuoka M, Yano S, Giaccone G et al. Final results from a phase II trial of ZD1839 ('Iressa') for patients with advanced non-small cell lung cancer (IDEAL 1). Proc Am Soc Clin Oncol 2002;22:298a.

4. Inoue A, Saijo Y, Maemondo M et al. Severe acute interstitial pneumonia and gefitinib. Lancet 2003;361:137-9.

5. Kris MG, Natale RB, Herbst RS et al. A phase II trial of ZD1839('Iressa') in advanced non-small cell lung cancer(NSCLC) patients who had failed platinumand docetaxel-based regimens(IDEAL 2). Proc Am Soc Clin Oncol 2002;22: 292a.

6. Villano JL, Mauer AM, Vokes EE. A case study documenting the anticancer activity of ZD1839(Iressa) in the brain. Ann Oncol 2003;14:656-8.

Submitted June 2003.

Accepted July 2003.

Hiroaki Satoh, M.D.,

University of Tsukuba, Institute of Clinical Medicine, Division of Respiratory Medicine, Tsukuba-city, Ibaraki, 305-8575,

Japan. e-mail: hirosato@md.tsukuba.ac.jp 